\title{
Evolution of star clusters moving on elliptic orbits
}

\author{
Holger Baumgardt \\ Dep. of Astronomy, School of Science, University of Tokyo, 7-3-1 \\ Hongo, Bunkyo-ko, Tokyo 113-0033, Japan
}

\begin{abstract}
We describe results of $N$-body simulations of star clusters moving on elliptic orbits. Our aim is to follow the evolution of these clusters and study how their dissolution times depend on particle number and eccentricity.

We find that the scaling of the lifetimes is nearly independent of the eccentricity, despite the fact that the amount of tidal shocking changes with eccentricity. In addition, no leveling off of the lifetimes at the high- $N$ end, which would indicate the influence of tidal shocking, is seen.
\end{abstract}

\section{The simulations}

The evolution of clusters containing between 1024 and 16384 equal-mass stars is followed using the fully collisional $N$-body code NBODY6++ (Spurzem \& Baumgardt 2001). Our clusters move on elliptic orbits around point-mass galaxies, starting at apogalacticon. We vary the eccentricity $e$ of the orbits between $e=0$ (circular orbits) and 0.9 . The density profiles of the clusters are given by King $W_{0}=3.0$ profiles and the tidal radii of the King profiles are initially adjusted such that they are equal to the tidal radii of the clusters at perigalacticon. We also perform runs with a Treecode to extend our results to higher $N$. In order to match them to the results of the fully collisional runs, test-runs are made which determine the amount of relaxation in the Treecode.

\section{Results}

We find that clusters on high eccentricity orbits lose mass only during their pericenter passages. After each pericenter passage, the tidal radius increases more quickly than the clusters expand due to relaxation and no stars are lost from the clusters.

Figure 1 shows the increase of the cluster lifetimes (defined as $4 \mathrm{x}$ the time taken to lose one quarter of the mass) with the particle number for two eccentricities. The lifetimes increase with increasing eccentricity since clusters spend a longer times in places with weak tidal fields. For a given eccentricity, the lifetimes increase close to $T_{D i s s} \sim t_{r h}^{2 / 3}$, where $t_{r h}$ is the half-mass relaxation time of the clusters. We find that the increase with the particle number is nearly independent of the eccentricity $e$, despite the fact that the amount of tidal shocking changes with eccentricity and there is no shocking in the circular case at all. 

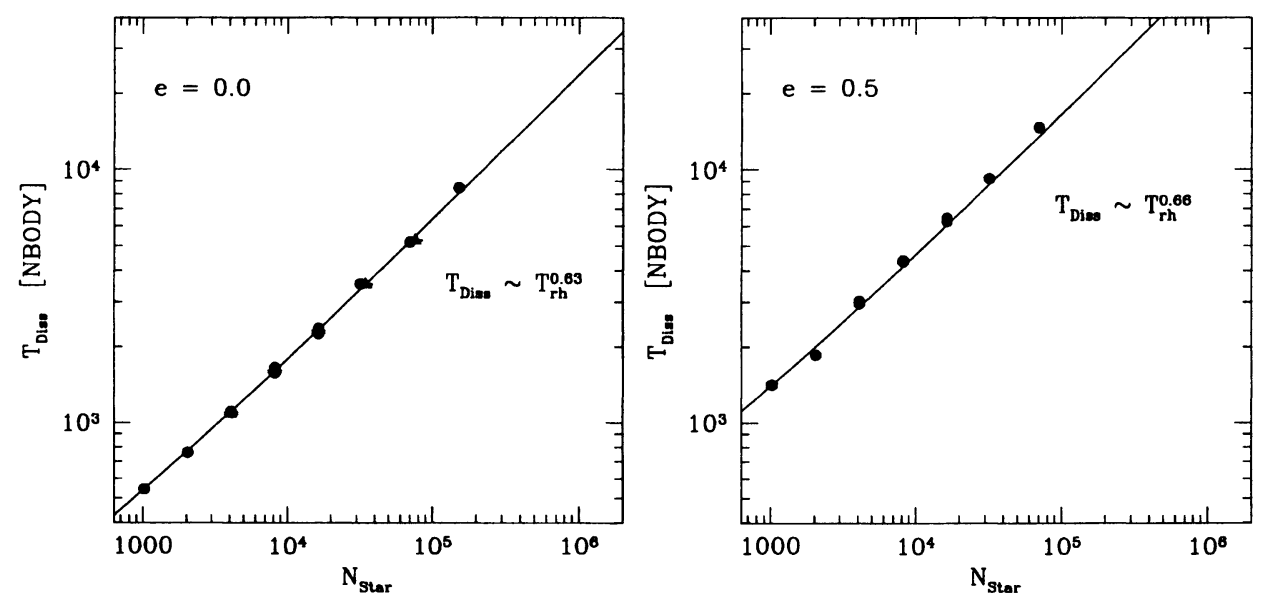

Figure 1. Lifetimes as a function of the particle number for a circular orbit and an orbit with eccentricity $e=0.5$. The lifetimes increase with increasing eccentricity due to the weaker tidal field, but the scaling with the particle number remains nearly the same.

The scaling law in the circular case can be explained by a combination of the long timescale that potential escapers need to leave the cluster and their backscattering towards lower energies (Baumgardt 2001). Muzzio et al. (2001) found that the equation of motion of stars on slightly elongated orbits can be approximated to first order by the motion on circular orbits that have the same angular momentum. This could explain the small amount of change in the scaling that we find, at least for orbits with small eccentricity.

\section{Conclusions}

The evolution of clusters moving on elliptic orbits and containing between 1024 and 16384 stars is studied. No bend in the lifetimes is found, indicating that tidal shocking is not the dominant escape mechanism for the particle range studied. We also find little change in the scaling with the particle number over a wide range of eccentricities. Both results indicate that relaxation is the important mass-loss mechanism. This could also be the case for globular clusters, since these have relaxation times comparable to those in our largest Treecode runs.

\section{References}

Baumgardt, H., 2001, MNRAS, 325, 1323

Muzzio, J.C. et al., 2001, private communication

Spurzem, R., Baumgardt, H., 2001, MNRAS submitted 